\title{
PROBLEMA DE SELEÇÃO DE FORNECEDORES: UMA ABORDAGEM UTILIZANDO ANALYTIC NETWORK PROCESS
}

\author{
Amanda Cecília Simões da Silva \\ Instituto Tecnológico de Aeronáutica - ITA \\ Pça Marechal E. Gomes, 50, Vila das Acácias, CEP 12228-900, São José dos Campos - SP \\ amanda.acsimoes@gmail.com \\ Mischel Carmen Neyra Belderrain \\ Instituto Tecnológico de Aeronáutica - ITA \\ Pça Marechal E. Gomes, 50, Vila das Acácias, CEP 12228-900, São José dos Campos - SP \\ carmen@ita.br
}

\section{RESUMO}

O Problema de Seleção de Fornecedores (SSP) é definido como o processo pelo qual os fornecedores são selecionados e avaliados para fazerem parte da cadeia de suprimentos. $\mathrm{O}$ objetivo deste trabalho é explorar a etapa de pré-qualificação do SSP, propondo a aplicação do método Analytic Network Process (ANP) com a abordagem ratings, definindo níveis de intensidade mínimos de desempenho em cada critério (limiares), de forma a reduzir as alternativas disponíveis para um conjunto menor de fornecedores aceitáveis. O método proposto foi aplicado num exemplo. Os resultados mostraram que a ordenação apresentada pelo método $\mathrm{ANP} /$ ratings, desconsiderando os limiares de cada critério selecionaria fornecedores "não aptos", devido a característica do método de permitir a compensação entre o desempenho dos critérios. Entretanto, com a definição de níveis de intensidade mínimo exigido de cada critério, foi possível realizar a triagem dos fornecedores realmente "aptos", para seguirem para a etapa de seleção final.

Palavra-chave: Pré-qualificação de fornecedores; Analytic Network Process; Ratings.

\begin{abstract}
The Supplier Selection Problem (SSP) is defined as the process by which suppliers are selected and evaluated to be part of the supply chain. The objective of this work is to explore the pre-qualification stage of the SSP, proposing the application of the Analytic Network Process (ANP) method with the ratings approach, defining minimum levels of performance intensity for each criterion (thresholds), in order to reduce the possible alternatives to a smaller set of acceptable suppliers. The proposed method was applied in an example. The results showed that the ordering presented by the ANP/ratings method, once you disregard the thresholds of each criterion would select "not suitable" suppliers, due to the characteristic of the method of allowing the compensation between the performance of the criteria. However, with the definition of minimum intensity levels required of each criterion,
\end{abstract}


it was possible to carry out the screening of the truly "suitable" suppliers, in order to proceed to the final selection stage.

Keywords: Supplier's pre-qualification; Analytic Network Process; Ratings.

\section{Como Citar:}

SILVA, A.C.S.; BELDERRAIN, M.C.N. Problema de Seleção de Fornecedores: Uma Abordagem Utilizando Analytic Network Process. In: SIMPÓSIO DE PESQUISA OPERACIONAL E LOGÍSTICA DA MARINHA, 19., 2019, Rio de Janeiro, RJ. Anais [...]. Rio de Janeiro: Centro de Análises de Sistemas Navais, 2019.

\section{INTRODUÇÃO}

O Problema de Seleção de Fornecedores (Supplier Selection Problem - SSP) consiste em analisar e medir o desempenho de um conjunto de fornecedores a fim de classificá-los e selecioná-los para melhorar a competitividade da cadeia de suprimentos (BRUNO et al., 2009). Tal problema é considerado complexo por possuir múltiplas alternativas e múltiplos critérios, tanto qualitativos e quantitativos, e que podem ser conflitantes entre si (SONMEZ, 2006).

A preocupação, com o tema SSP, é evidenciada na literatura desde a década de 60, quando Dickson (1966) identificou os principais critérios envolvidos no processo de seleção. Desde então, vários trabalhos sobre revisões de literatura vêm sendo publicadas, dentre as principais citam-se: (DEBOER et al., 2001), (BHUTTA, 2003), (SONMEZ, 2006), (AISSAOIU et al., 2007), (HO et al., 2010), (AGARWAL et al., 2011), (WARE et al., 2012), (GENOVESE et al., 2013), (CHAI et al., 2013), (GOVINDAN et al., 2015), (YILDIZ e YAYLA 2015), (ASSELAOU et al., 2015), (HAMDI et al., 2015), (WETZSTEIN et al., 2016), (SIMIC et al., 2017), (OCAMPO et al., 2018), entre outros.

No entanto, estas revisões de literatura variam em áreas de interesse. Ho et al. (2010) estudou 78 artigos de periódicos internacionais de 2000 a 2008 e classificou-os quanto aos critérios de avaliação mais utilizados e abordagens de seleção mais populares (HO et al., 2010).

Genovese et al. (2013) e Govindan et al. (2015) focaram suas revisões de literatura em seleção de fornecedores verdes, onde fatores ambientais são incorporados no SSP, devido a crescente preocupação ambiental nas operações das empresas. Ainda segundo Govindan et al. (2015) os resultados mostram que, embora exista um interesse crescendo sobre fatores ambientais dentro da literatura de SSP, ainda há um número relativo baixo de artigos publicados que incorporam critérios ambientais, quando comparado com o grande volume de publicações referente apenas ao tema SSP (GENOVESE et al., 2013; GOVINDAN et al., 2015).

Ocampo et al. (2018) analisaram 244 artigos que utilizaram várias abordagens de seleção de fornecedores no período de 2006 a 2016. As abordagens identificadas foram classificadas em: 1) abordagens individuais (métodos MCDM - Multiple Criteria Decision Making), programação matemática, inteligência artificial, abordagens de decisão fuzzy e modelagem estatística), 2) abordagens integradas, e; 3) abordagens emergentes (abordagens que não são amplamente conhecidas e fornecem uma nova maneira de analisar o problema de seleção de fornecedores). A revisão realizada pelos autores revela que $61 \%$ dos artigos 
analisados empregam abordagens integradas, 31\% aplicam abordagens individuais e $8 \%$ abordagens emergentes. A abordagem fuzzy integrada é mais adotada dentro das abordagens integradas, e Programação matemática é a mais adotada dentro das categorias de abordagens individuais (OCAMPO et al., 2018).

DeBoer et al. (2001) apresentam o desenvolvimento do SSP em quatro etapas: Etapa 1: Definição do problema; Etapa 2: Formulação dos critérios de decisão; Etapa 3: Préqualificação dos fornecedores potenciais; e, Etapa 4: Seleção final dos fornecedores (DEBOER et al., 2001). Sonmez (2006) considera mais uma etapa adicional neste processo: a de Monitoramento dos fornecedores selecionados, denominada Etapa 5 (SONMEZ, 2006),

As últimas revisões de literatura, sobre técnicas utilizadas no SSP, mostram que a etapa de seleção final (etapa 4) concentra a vasta maioria de publicações existentes na literatura (OCAMPO et al., 2018), (SIMIC et al., 2017), (YILDIZ e YAYLA 2015), (CHAI et al., 2013). Poucos trabalhos exploram a etapa de pré-qualificação (etapa 3) (SARKAR e MOHAPATRA, 2006), (GALO e CARPINETTI, 2018), (LIMA JUNIOR et al., 2013), (SEPULVEDA e DERPICH, 2015), (ARAZ e OZKARAHAN, 2007). Todos estes autores utilizaram métodos de classificação para esta etapa.

Araz e Ozkarahan (2007) utilizam métodos multicritério da família PROMÉTHÉE (Preference Ranking Organization Method for Enrichment Evalutions) e suas variações (Prométhée sorting) para a etapa 3 (ARAZ e OZKARAHAN, 2007). E mais recentemente Galo e Carpinetti (2018) propõem a utilização de um modelo de apoio à tomada de decisão que combina o ELECTRE TRI e os conjuntos Triangular Hesitant Fuzzy (GALO e CARPINETTI, 2018).

Apesar de todas as etapas do SSP serem importantes, este trabalho propõe explorar a etapa de pré-qualificação (etapa 3), propondo a aplicação de um método de ordenação, o Analytic Network Process (ANP) com a abordagem ratings, definindo níveis de intensidade mínimos de desempenho em cada critério, de forma a reduzir as alternativas disponíveis para um conjunto menor de fornecedores aceitáveis.

\section{ANALYTIC NETWORK PROCESS (ANP) COM ABORDAGEM RATINGS}

Desenvolvido por Thomas L. Saaty, o ANP é um método de apoio multicritério a decisão, considerado uma generalização do Analytic Hierarchy Process (AHP). Dessa maneira, o ANP utiliza uma rede (ao invés de hierarquia, como no AHP), não sendo necessário especificar níveis, além de permitir relações de dependência entre seus elementos ou nós (SAATY e OZDEMIR, 2005).

Segundo Saaty (2006) existem duas formas de realizar as comparações par a par utilizadas nos métodos AHP e ANP, são elas: a) por meio de comparações em termos de medição absoluta (do inglês, absolute measurement); e, b) por meio de comparações em termos de medição relativa (do inglês, relative measurement). Na medição absoluta (também conhecida como ratings) as alternativas são avaliadas de acordo com os níveis de intensidade obtidos de cada atributo (critérios e subcritérios no AHP e nós no ANP). Na medição relativa, as alternativas são comparadas par a par à luz de um critério.

Conforme mencionado, a medição absoluta (ratings) tem a vantagem de estabelecer padrões que as alternativas devem cumprir. Ou seja, o decisor, caso queira, pode atribuir um nível mínimo aceitável (limiar) dos níveis de intensidade de cada atributo, conforme esquematizado na Figura 1. Esse tipo de atribuição serve para verificar se alguma alternativa não atende o requisito mínimo de desempenho em algum critério/subcritério/nó. 
Figura 1 - Limiares dos níveis de intensidade dos critérios para a avaliação de alternativas.

Critério $\mathrm{C}_{1}$ Critério $\mathrm{C}_{2}$ Critério $\mathrm{C}_{3}$ Critério $\mathrm{C}_{\mathrm{n}}$

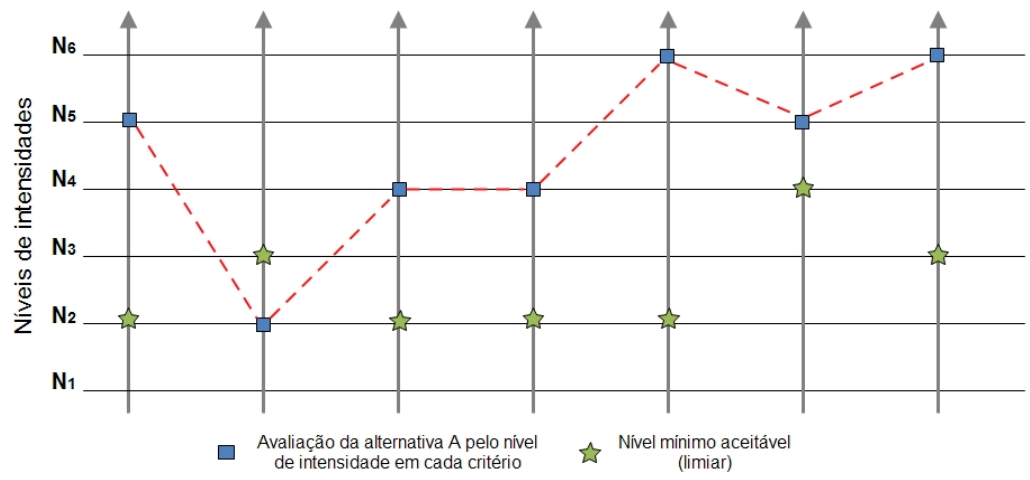

Silva (2012) apresenta três etapas para aplicação do método ANP com a abordagem ratings: 1) formulação do problema, 2) julgamentos e 3) estruturação da supermatriz e obtenção dos resultados, conforme mostrado na Figura 2.

Figura 2 - Etapas do método ANP com a abordagem ratings.

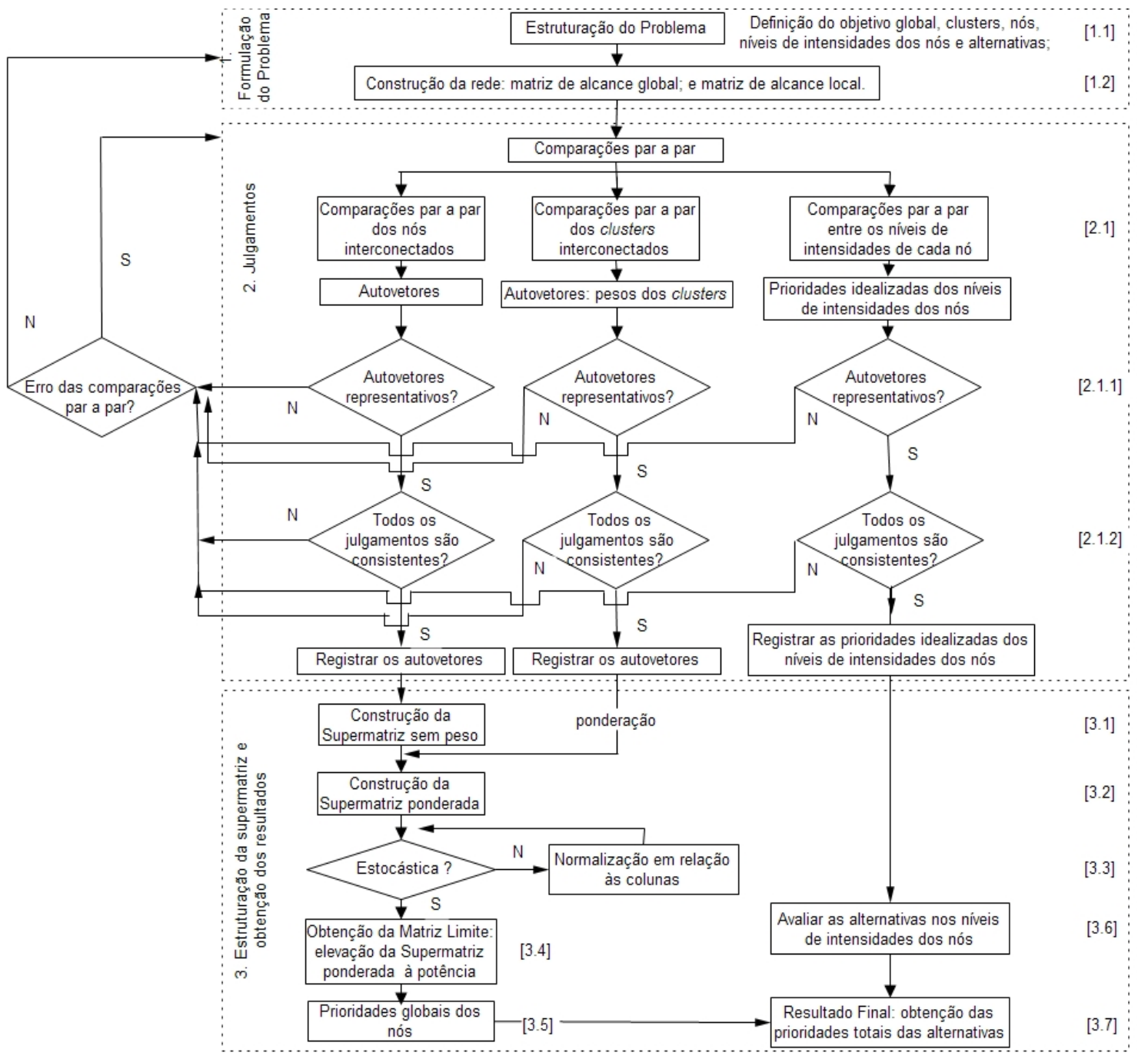


Na Etapa 1 (Formulação do Problema) define-se o objetivo do processo decisório, os clusters, critérios, denominado nós na aplicação do ANP, com seus respectivos níveis de intensidade $\mathrm{e}$ as alternativas. Posteriormente constrói-se a rede de clusters com seus respectivos critérios (nós) e estabelecem-se as relações de dependência e feedback entre eles. Inserem-se os níveis de intensidade nos critérios (nós). A rede não possui o cluster das alternativas, porém estas são inseridas internamente na rede. As alternativas são classificadas conforme os níveis de intensidade dos nós, ou seja, para cada alternativa são atribuídos os níveis de intensidade de cada critério (nó) da rede.

Para este trabalho definiu-se o limiar (nível mínimo aceitável) dos níveis de intensidade de cada nó.

$\mathrm{Na}$ Etapa 2 (Julgamentos) as comparações par a par são realizadas para todas as conexões existentes na rede e também entre os níveis de intensidade de cada nó, de acordo com a Escala Fundamental de Saaty. As comparações par a par dividem-se em três casos: 1) comparações par a par entre os nós interconectados; 2) comparações par a par entre os clusters interconectados; e, 3) comparações par a par entre os níveis de intensidade de cada nó. Uma vez realizadas todas as comparações e verificada a consistência dos julgamentos são registrados os autovetores e as prioridades idealizadas dos níveis de intensidade (valores numéricos dos ratings).

Na Etapa 3 (Estruturação da supermatriz e obtenção dos resultados) são construídas as supermatrizes (sem pesos, ponderada e limite) e são obtidas as prioridades globais dos nós. Posteriormente as alternativas são classificadas nos respectivos níveis de intensidade de cada nó, obtendo assim as prioridades locais das alternativas. Por fim, obtêm-se as prioridades totais das alternativas, agregando as prioridades globais dos nós com os valores numéricos idealizados dos níveis de intensidade em cada nó (prioridades locais das alternativas).

Maiores detalhes sobre as etapas e passos do método ANP com a abordagem ratings (SILVA, 2012).

A próxima seção apresenta um exemplo de aplicação do método ANP com a abordagem ratings para a etapa de pré-qualificação do SSP.

\section{APLICAÇÃO DO MÉTODO ANP COM A ABORDAGEM RATINGS NA ETAPA DE PRÉ-QUALIFICAÇÃO DO SSP}

O contexto do exemplo é o seguinte: A Empresa A, com mais de 40 anos no mercado, é uma das maiores fabricantes de motores automotivos do Brasil. São inúmeros os itens produzidos pela empresa, mas alguns destes são adquiridos de fornecedores. Um deles é o filtro de óleo, item importante para preservar a vida útil do motor. Para este produto, existem 20 fornecedores conhecidos. Os decisores neste exemplo são os autores do trabalho. A decisão é identificar quais fornecedores são aptos ou não aptos para o fornecimento do produto.

O método ANP com a abordagem ratings foi aplicado com o auxílio do software SuperDecisions (www.superdecisions.com), seguindo as etapas e passos apresentados na Figura 2. A seguir são apresentados somente as principais saídas de cada uma das etapas.

\subsection{ETAPA 1: FORMULAÇÃO DO PROBLEMA DE DECISÃO}

A definição dos critérios no SSP geralmente surge a partir dos aspectos que as empresas levam em conta na seleção de fornecedores e os mesmos serão usados para comparar as alternativas. Portanto os critérios variam de acordo com as características e necessidade de cada organização, do contexto de decisão e dos stakeholders envolvidos. 
Para o exemplo em questão foram considerados sete critérios (nós) apresentados na revisão de literatura de Ho et al. (2010). A Tabela 1 apresenta a definição de cada critério.

Tabela 1 - Critérios (nós) estabelecidos e suas respectivas descrições.

\begin{tabular}{c|l}
\hline Critérios & \multicolumn{1}{c}{ Definição } \\
\hline Qualidade & $\begin{array}{l}\text { Avaliado em termos de o fornecedor oferecer um produto durável e } \\
\text { confiável dentro das conformidades e especificações da empresa. }\end{array}$ \\
\hline Entrega & $\begin{array}{l}\text { Avalia a quantidade de entregas de transporte que foram efetivamente } \\
\text { entregues no prazo acordado em contrato e a conformidade de entrega. }\end{array}$ \\
\hline Custo & $\begin{array}{l}\text { Avaliado em termo de custo-benefício, o quanto é justo pagar por um } \\
\text { produto ou serviço que trará um rendimento melhor para a empresa. }\end{array}$ \\
\hline $\begin{array}{c}\text { Competência } \\
\text { da Produção }\end{array}$ & $\begin{array}{l}\text { Consiste em avaliar vários pontos como: equipe de trabalho bem } \\
\text { treinada e flexível, máquinas, equipamentos, sistemas e tecnologias } \\
\text { atualizados, dentre outros. }\end{array}$ \\
\hline Serviço & $\begin{array}{l}\text { Avaliado com base na flexibilidade da produção do fornecedor e na } \\
\text { flexibilidade de entrega. }\end{array}$ \\
\hline Reputação & $\begin{array}{l}\text { Avaliado por meio do histórico e recomendações de outras empresas } \\
\text { que já usufruíram dos mesmos produtos ou serviço. }\end{array}$ \\
\hline Riscos & $\begin{array}{l}\text { Possíveis perdas em longo prazo. Avaliado por meio do histórico, } \\
\text { entrega no prazo, estabilidade financeira e flutuação dos custos. }\end{array}$ \\
\hline
\end{tabular}

A Tabela 2 apresenta os níveis de intensidade (ratings) dos critérios (nós). O limiar de cada nível de intensidade é apresentado em negrito.

Tabela 2 - Níveis de intensidade dos nós.

\begin{tabular}{l|l}
\hline \multicolumn{1}{c|}{ Critérios (nós) } & \multicolumn{1}{c}{ Níveis de intensidade } \\
\hline Qualidade & Excelente, Muito bom, Bom, Regular e Ruim \\
\hline Entrega & Dentro do prazo, Pouco atraso e Muito atraso \\
\hline Custo & Excelente, Muito bom, Bom, Regular e Ruim \\
\hline Competência da Produção & Competente, Regular e Baixa competência \\
\hline Serviço & $\begin{array}{l}\text { Nível de serviço alto, Nível de serviço médio e Nível de } \\
\text { serviço baixo }\end{array}$ \\
\hline Reputação & Excelente reputação, Boa reputação e Baixa reputação \\
\hline Riscos & Alto, Médio, Baixo \\
\hline
\end{tabular}

As alternativas de decisão inicialmente consideradas são os 20 fornecedores de filtro de óleo. Cada fornecedor foi avaliado com base nas definições dos nós apresentados na Tabela 1 e classificados de acordo com os níveis de intensidade apresentados na Tabela 2 . A Tabela 3 apresenta os fornecedores com seus respectivos níveis de intensidade em cada nó. Observa-se que alguns fornecedores possuem níveis de intensidade abaixo do limiar, estipulado pelos decisores, e está em negrito sublinhado na Tabela 3. 
Tabela 3 - Alternativas e seus níveis de intensidade em cada critério (nó).

\begin{tabular}{|c|c|c|c|c|c|c|c|}
\hline & Qualidade & Entrega & Custo & Competência & Serviço & Reputação & Riscos \\
\hline Fornec. 1 & Muito bom & Dentro do prazo & Muito bom & Regular & Alto & Excelente & Baixo \\
\hline Fornec. 2 & Muito bom & Pouco atraso & Bom & Regular & Médio & Boa & Médio \\
\hline Fornec. 3 & Excelente & Dentro do prazo & Regular & Competente & Alto & Boa & Médio \\
\hline Fornec. 4 & Muito bom & Dentro do prazo & Muito bom & Competente & Alto & Excelente & Baixo \\
\hline Fornec. 5 & Muito bom & Dentro do prazo & Muito bom & Regular & Médio & Boa & Médio \\
\hline Fornec. 6 & Bom & Pouco atraso & Bom & Regular & Médio & Boa & Médio \\
\hline Fornec. 7 & Muito bom & Dentro do prazo & Excelente & Competente & Alto & Excelente & Baixo \\
\hline Fornec. 8 & Muito bom & Pouco atraso & Muito bom & Regular & Médio & Boa & Médio \\
\hline Fornec. 9 & Bom & Dentro do prazo & Bom & Regular & Médio & Boa & Médio \\
\hline Fornec. 10 & Regular & Pouco atraso & Muito bom & Competente & Médio & Baixa & Alto \\
\hline Fornec. 11 & Excelente & Pouco atraso & Bom & Competente & Médio & Excelente & Médio \\
\hline Fornec. 12 & Excelente & Pouco atraso & Excelente & Competente & Alto & Excelente & Médio \\
\hline Fornec. 13 & Bom & Muito atraso & Bom & Regular & Baixo & Boa & Alto \\
\hline Fornec. 14 & Excelente & Pouco atraso & Muito bom & Competente & Alto & Excelente & Baixo \\
\hline Fornec. 15 & Excelente & Pouco atraso & Muito bom & Competente & Médio & Excelente & Baixo \\
\hline Fornec. 16 & Regular & Dentro do prazo & Bom & Baixa & Baixo & Baixa & Médio \\
\hline Fornec. 17 & Muito bom & Dentro do prazo & Bom & Competente & Médio & Boa & Baixo \\
\hline Fornec. 18 & Excelente & Pouco atraso & Bom & Competente & Alto & Excelente & Médio \\
\hline Fornec. 19 & Bom & Dentro do prazo & Muito bom & Regular & Médio & Boa & Médio \\
\hline Fornec. 20 & Bom & Pouco atraso & Bom & Competente & Alto & Boa & Médio \\
\hline
\end{tabular}

A Figura 3 apresenta a rede para o exemplo de pré-qualificação de fornecedores. Observe o cluster do Objetivo e o cluster de Critérios (nós).

Figura 3 - Rede do exemplo de seleção de fornecedores.

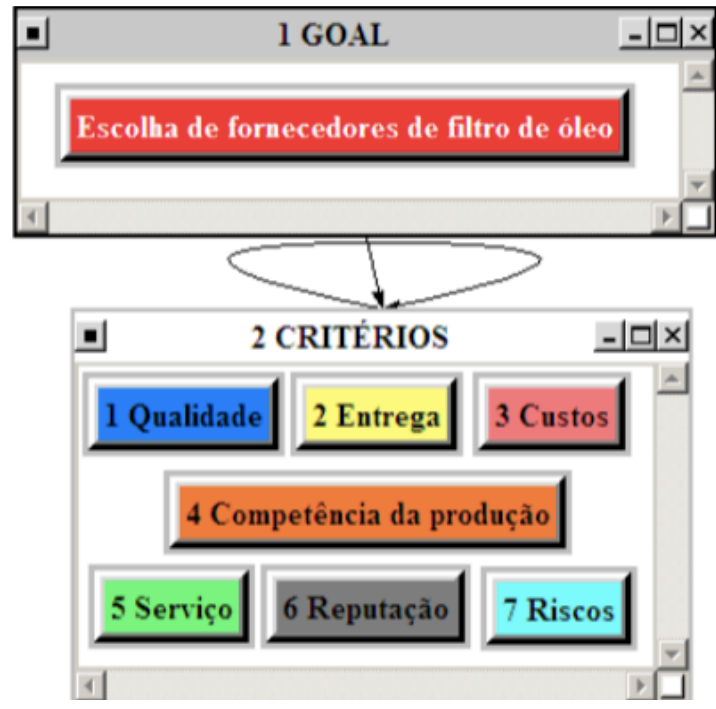

A Tabela 4 apresenta a matriz de alcance local que especifica a relação de dependência entre os nós do cluster critério, representado pelo loop da Figura 3. O valor 1 é atribuído sempre que houver dependência. Caso contrário, atribui-se o valor zero.

Por exemplo, observando a primeira coluna da matriz de alcance local, o critério Qualidade influencia os critérios Entrega, Custo, Competência da produção e Reputação, representado pelo valor 1 . 
Tabela 4 - Matriz de alcance local para o exemplo de seleção de fornecedores.

\begin{tabular}{l|c|c|c|c|c|c|c}
\hline & Q & E & C & CP & S & Rep & R \\
\hline Qualidade (Q) & 0 & 0 & 1 & 1 & 0 & 0 & 1 \\
\hline Entrega (E) & 1 & 0 & 1 & 1 & 1 & 0 & 1 \\
\hline Custo (C) & 1 & 1 & 0 & 1 & 1 & 0 & 1 \\
\hline Competência (CP) & 1 & 0 & 1 & 0 & 1 & 1 & 0 \\
\hline Serviço (S) & 0 & 1 & 1 & 0 & 0 & 1 & 1 \\
\hline Reputação (Rep) & 1 & 1 & 0 & 1 & 1 & 0 & 1 \\
\hline Riscos (R) & 0 & 0 & 1 & 0 & 0 & 1 & 0 \\
\hline
\end{tabular}

Após a construção da rede (Figura 3) e da matriz de alcance local (Tabela 4), procede-se a etapa de julgamentos.

\subsection{ETAPA 2: JULGAMENTOS}

Todas as comparações par a par foram realizadas utilizando a Escala Fundamental de Saaty (SAATY, 2006). A Figura 4 (a) apresenta a matriz de comparação par a par dos níveis de intensidade para o critério (nó) Reputação do cluster critérios. Os níveis de intensidade adotados para este critério são: excelente reputação, boa reputação e baixa reputação (Tabela 2). A Figura 4 (b) e (c) apresentam o vetor de prioridades obtido por meio do cálculo do autovetor direito e o vetor de prioridades idealizado obtido atribuindo-se a melhor categoria o valor igual a 1 e as outras proporcionalmente menor, respectivamente. Procedeu-se de forma análoga para os demais critérios (nós) da rede.

Figura 4 - Vetor de prioridades em valores numéricos dos níveis de intensidade para o nó reputação do cluster critérios.

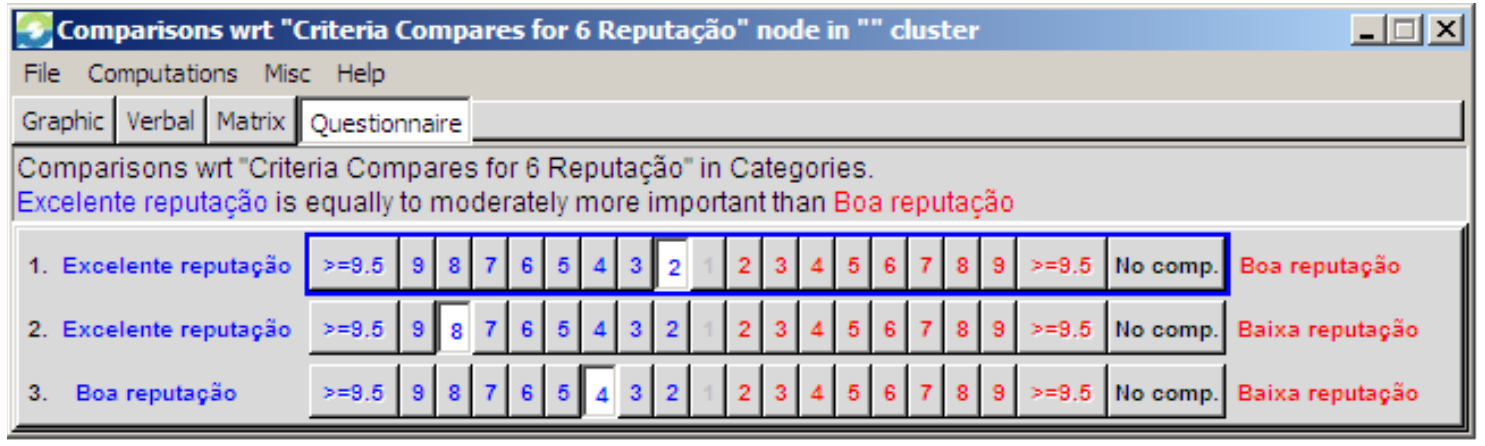

(a) Matriz de comparação: comparações par a par entre os níveis de intensidade do nó reputação do cluster critérios.

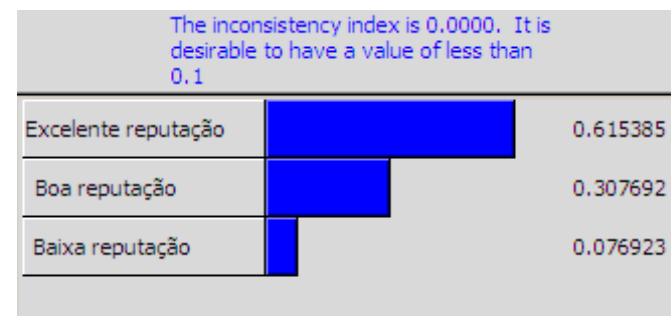

(b) Vetor de prioridades.

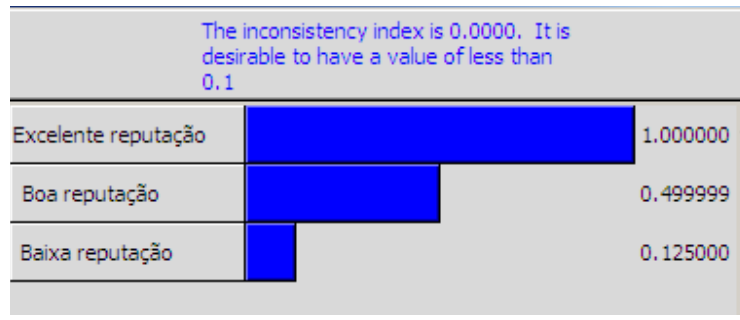

(c) Vetor de prioridades idealizado. 
De acordo com os decisores, todos os autovetores obtidos refletem o problema assim como todos os valores da Razão de Consistência (RC) obtidos das matrizes de comparação estão dentro do limite aceitável $(\mathrm{RC}<0,10)$. Desta forma, os autovetores e as prioridades idealizadas dos níveis de intensidade (valores numéricos dos níveis de intensidade) são registrados.

\subsection{ETAPA 3: ESTRUTURAÇÃO DA SUPERMATRIZ E OBTENÇÃO DOS RESULTADOS}

As prioridades globais dos nós são oriundas da matriz limite. Representam os "pesos" de cada nó. As prioridades globais foram: Qualidade $(0,230)$, Entrega $(0,143)$, Custo $(0,334)$, Competência da produção $(0,160)$, Serviço $(0,073)$, Reputação $(0,044)$ e Riscos $(0,016)$. Os nós Custo $(0,334)$ e Qualidade $(0,230)$ apresentaram as maiores prioridades do cluster critérios para a aplicação em curso.

Nesta etapa as alternativas são avaliadas nos níveis de intensidade. O desempenho dos fornecedores são inseridos no modelo, conforme apresentado na Tabela 5.

Tabela 5 - Classificação das alternativas nos níveis de intensidade dos critérios.

\begin{tabular}{|c|c|c|c|c|c|c|c|c|}
\hline & Priorities & $\begin{array}{l}1 \text { Qualidade } \\
0.230475\end{array}$ & $\begin{array}{l}\text { 2 Entrega } \\
0.143254\end{array}$ & $\begin{array}{l}3 \text { Custos } \\
0.333569\end{array}$ & $\begin{array}{l}4 \text { Competência da h } \\
0.159549\end{array}$ & \begin{tabular}{|l}
5 Servico \\
0.073194
\end{tabular} & $\begin{array}{l}\text { 6 Reputação } \\
0.043550\end{array}$ & $\begin{array}{l}\text { 7 Riscos } \\
0.016410\end{array}$ \\
\hline Fornecedor 1 & 0.060618 & Muito bom & Dentro do prazo & Muito bom & Regular & Nível de serviço alto & Excelente reputação & Baixo \\
\hline Fornecedor 2 & 0.036957 & Muito bom & Pouco atraso & Bom & Regular & Nivel de serviço médio & Boa reputação & Médio \\
\hline Fornecedor 3 & 0.054096 & Excelente & Dentro do prazo & Regular & Competente & Nivel de serviço alto & Boa reputação & Médio \\
\hline Fornecedor 4 & 0.068585 & Muito bom & Dentro do prazo & Muito bom & Competente & Nivel de serviço alto & Excelente reputação & Baixo \\
\hline Fornecedor 5 & 0.054621 & Muito bom & Dentro do prazo & Muito bom & Regular & Nivel de serviço médio & Boa reputação & Médio \\
\hline Fornecedor 6 & 0.029423 & Bom & Pouco atraso & Bom & Regular & Nivel de serviço médio & Boa reputação & Médio \\
\hline Fornecedor 7 & 0.075072 & Muito bom & Dentro do prazo & Excelente & Competente & Nivel de serviço alto & Excelente reputação & Baixo \\
\hline Fornecedor 8 & 0.047788 & Muito bom & Pouco atraso & Muito bom & Regular & Nivel de serviço médio & Boa reputação & Médio \\
\hline Fornecedor 9 & 0.036256 & Bom & Dentro do prazo & Bom & Regular & Nivel de serviço médio & Boa reputação & Médio \\
\hline Fornecedor 10 & 0.042517 & Regular & Pouco atraso & Muito bom & Competente & Nivel de serviço médio & Baixa reputação & Alto \\
\hline Fornecedor 11 & 0.050442 & Excelente & Pouco atraso & Bom & Competente & Nivel de serviço médio & Excelente reputação & Médio \\
\hline Fornecedor 12 & 0.071414 & Excelente & Pouco atraso & Excelente & Competente & Nível de serviço alto & Excelente reputação & Médio \\
\hline Fornecedor 13 & 0.023950 & Bom & Muito atraso & Bom & Regular & Nível de serviço baixo & Boa reputação & Alto \\
\hline Fornecedor 14 & 0.065551 & Excelente & Pouco atraso & Muito bom & Competente & Nível de serviço alto & Excelente reputação & Baixo \\
\hline Fornecedor 15 & 0.061897 & Excelente & Pouco atraso & Muito bom & Competente & Nivel de serviço médio & Excelente reputação & Baixo \\
\hline Fornecedor 16 & 0.026202 & Regular & Dentro do prazo & Bom & Baixa competência & Nivel de serviço baixo & Baixa reputação & Médio \\
\hline Fornecedor 17 & 0.052381 & Muito bom & Dentro do prazo & Bom & Competente & Nivel de serviço médio & Boa reputação & Baixo \\
\hline Fornecedor 18 & 0.054097 & Excelente & Pouco atraso & Bom & Competente & Nível de serviço alto & Excelente reputação & Médio \\
\hline Fornecedor 19 & 0.047087 & Bom & Dentro do prazo & Muito bom & Regular & Nivel de serviço médio & Boa reputação & Médio \\
\hline Fornecedor 20 & 0.041045 & Bom & Pouco atraso & Bom & Competente & Nivel de serviço alto & Boa reputação & Médio \\
\hline
\end{tabular}

A Tabela 6 apresenta os valores numéricos dos níveis de intensidade dos critérios, cujo processo foi explicado na Figura 4. Por exemplo, para o nó Reputação, os seus níveis de intensidade excelente reputação, boa reputação e baixa reputação recebem os valores $1,00,0,5$ e 0,125 , respectivamente. 
Tabela 6 - Valores numéricos dos níveis de intensidade dos critérios.

\begin{tabular}{|c|c|c|c|c|c|c|c|c|}
\hline & Priorities & $\begin{array}{l}1 \text { Qualidade } \\
0.230475\end{array}$ & $\begin{array}{l}\text { 2 Entrega } \\
0.143254\end{array}$ & $\begin{array}{l}3 \text { Custos } \\
0.333569\end{array}$ & \begin{tabular}{|l|}
4 Competência da \\
0.159549
\end{tabular} & $\begin{array}{l}5 \text { Serviço } \\
0.073194\end{array}$ & $\begin{array}{l}\text { 6 Reputação } \\
0.043550\end{array}$ & $\begin{array}{l}\text { 7 Riscos } \\
0.016410\end{array}$ \\
\hline Fornecedor 1 & 0.060618 & 0.790948 & 1.000000 & 0.753435 & 0.366884 & 1.000000 & 1.000000 & 1.000000 \\
\hline Fornecedor 2 & 0.036957 & 0.790948 & 0.395212 & 0.341757 & 0.366884 & 0.366884 & 0.500001 & 0.517875 \\
\hline Fornecedor 3 & 0.054096 & 1.000000 & 1.000000 & 0.147300 & 1.000000 & 1.000000 & 0.500001 & 0.517875 \\
\hline Fornecedor 4 & 0.068585 & 0.790948 & 1.000000 & 0.753435 & 1.000000 & 1.000000 & 1.000000 & 1.000000 \\
\hline Fornecedor 5 & 0.054621 & 0.790948 & 1.000000 & 0.753435 & 0.366884 & 0.366884 & 0.500001 & 0.517875 \\
\hline Fornecedor 6 & 0.029423 & 0.376492 & 0.395212 & 0.341757 & 0.366884 & 0.366884 & 0.500001 & 0.517875 \\
\hline Fornecedor 7 & 0.075072 & 0.790948 & 1.000000 & 1.000000 & 1.000000 & 1.000000 & 1.000000 & 1.000000 \\
\hline Fornecedor 8 & 0.047788 & 0.790948 & 0.395212 & 0.753435 & 0.366884 & 0.366884 & 0.500001 & 0.517875 \\
\hline Fornecedor 9 & 0.036256 & 0.376492 & 1.000000 & 0.341757 & 0.366884 & 0.366884 & 0.500001 & 0.517875 \\
\hline Fornecedor 10 & 0.042517 & 0.162807 & 0.395212 & 0.753435 & 1.000000 & 0.366884 & 0.125001 & 0.107277 \\
\hline Fornecedor 11 & 0.050442 & 1.000000 & 0.395212 & 0.341757 & 1.000000 & 0.366884 & 1.000000 & 0.517875 \\
\hline Fornecedor 12 & 0.071414 & 1.000000 & 0.395212 & 1.000000 & 1.000000 & 1.000000 & 1.000000 & 0.517875 \\
\hline Fornecedor 13 & 0.023950 & 0.376492 & 0.093714 & 0.341757 & 0.366884 & 0.100951 & 0.500001 & 0.107277 \\
\hline Fornecedor 14 & 0.065551 & 1.000000 & 0.395212 & 0.753435 & 1.000000 & 1.000000 & 1.000000 & 1.000000 \\
\hline Fornecedor 15 & 0.061897 & 1.000000 & 0.395212 & 0.753435 & 1.000000 & 0.366884 & 1.000000 & 1.000000 \\
\hline Fornecedor 16 & 0.026202 & 0.162807 & 1.000000 & 0.341757 & 0.100951 & 0.100951 & 0.125001 & 0.517875 \\
\hline Fornecedor 17 & 0.052381 & 0.790948 & 1.000000 & 0.341757 & 1.000000 & 0.366884 & 0.500001 & 1.000000 \\
\hline Fornecedor 18 & 0.054097 & 1.000000 & 0.395212 & 0.341757 & 1.000000 & 1.000000 & 1.000000 & 0.517875 \\
\hline Fornecedor 19 & 0.047087 & 0.376492 & 1.000000 & 0.753435 & 0.366884 & 0.366884 & 0.500001 & 0.517875 \\
\hline Fornecedor 20 & 0.041045 & 0.376492 & 0.395212 & 0.341757 & 1.000000 & 1.000000 & 0.500001 & 0.517875 \\
\hline
\end{tabular}

A Figura 5, apresenta de forma gráfica, a pontuação final para cada alternativa (fornecedor), representada pela coluna "Total".

No momento da análise dos resultados pelo decisor, o mesmo deve atentar para o valor da coluna "Total" da Figura 5, pois quanto mais próximo de 1 este valor, maior o desempenho do fornecedor nos critérios. Por exemplo o fornecedor 7, com prioridade total de 0,9518, foi classificado apto por possuir os melhores níveis de intensidade em cada critério, como pode ser visto nas Tabela 5 e 6.

$\mathrm{O}$ decisor deve também se atentar ao fato que o método ANP utiliza regras compensatórias, ou seja um fornecedor com prioridade total alta pode não garantir um nível mínimo de desempenho em algum critério. É o caso do Fornecedor 3, que apesar de ter obtido uma prioridade total de 0,6859, deve ser eliminado, pois apresentou nível de intensidade no critério Custo abaixo do limiar estipulado (regular), conforme Tabela 5. Situação análoga ocorre com os fornecedores 10, 13 e 16. Por exemplo o fornecedor 10, décimo quarto colocado, apresenta níveis de intensidade abaixo do limiar estipulado nos critérios Qualidade, Reputação e Riscos.

Neste exemplo foram adotados liminares para os critérios, de forma a classificar o fornecedor como não apto, caso não atenda ao limiar mínimo estipulado. O ranking final dos fornecedores e sua classificação são apresentados na penúltima e última coluna da Figura 5, respectivamente.

Diante da situação os fornecedores 3, 10, 13 e 16 foram classificados como fornecedores não aptos e o restante como fornecedores aptos, por atenderem aos requisitos mínimos de desempenho em todos os critérios. 
Figura 5 - Pontuação final, ranking e classificação das alternativas.

\begin{tabular}{|c|c|c|c|c|c|c|}
\hline Graphic & Ratings Alternatives & Total & Ideal & Normal & Ranking & Classificação \\
\hline & Fornecedor 1 & 0.7686 & 0.8075 & 0.0606 & 6 & Apto \\
\hline & Fornecedor 2 & 0.4686 & 0.4923 & 0.0370 & 16 & Apto \\
\hline & Fornecedor 3 & 0.6859 & 0.7206 & 0.0541 & 9 & Não apto \\
\hline & Fornecedor 4 & 0.8696 & 0.9136 & 0.0686 & 3 & Apto \\
\hline & Fornecedor 5 & 0.6925 & 0.7276 & 0.0546 & 7 & Apto \\
\hline & Fornecedor 6 & 0.3730 & 0.3919 & 0.0294 & 18 & Apto \\
\hline & Fornecedor 7 & 0.9518 & 1.0000 & 0.0751 & 1 & Apto \\
\hline & Fornecedor 8 & 0.6059 & 0.6366 & 0.0478 & 12 & Apto \\
\hline & Fornecedor 9 & 0.4597 & 0.4830 & 0.0363 & 17 & Apto \\
\hline & Fornecedor 10 & 0.5391 & 0.5664 & 0.0425 & 14 & Não apto \\
\hline & Fornecedor 11 & 0.6395 & 0.6719 & 0.0504 & 11 & Apto \\
\hline & Fornecedor 12 & 0.9055 & 0.9513 & 0.0714 & 2 & Apto \\
\hline & Fornecedor 13 & 0.3037 & 0.3190 & 0.0239 & 20 & Não apto \\
\hline & Fornecedor 14 & 0.8311 & 0.8732 & 0.0656 & 4 & Apto \\
\hline & Fornecedor 15 & 0.7848 & 0.8245 & 0.0619 & 5 & Apto \\
\hline & Fornecedor 16 & 0.3322 & 0.3490 & 0.0262 & 19 & Não apto \\
\hline & Fornecedor 17 & 0.6641 & 0.6978 & 0.0524 & 10 & Apto \\
\hline & Fornecedor 18 & 0.6859 & 0.7206 & 0.0541 & 8 & Apto \\
\hline & Fornecedor 19 & 0.5970 & \begin{tabular}{|l|}
0.6272 \\
\end{tabular} & 0.0471 & 13 & Apto \\
\hline & Fornecedor 20 & 0.5204 & 0.5467 & 0.0410 & 15 & Apto \\
\hline
\end{tabular}

Uma vez garantido níveis mínimos de desempenho em cada critério na avaliação dos fornecedores, o decisor poderá selecionar dentre os fornecedores classificados como aptos aqueles com maiores prioridades totais, para seguirem para a etapa de seleção final.

\section{ANÁLISE DOS RESULTADOS}

Este trabalho focou na Etapa 3 (pré-qualificação dos fornecedores potenciais) do SSP. O método ANP com abordagem ratings foi aplicado para realizar o ranking de 20 fornecedores, utilizando-se de sete critérios (nós) para esta seleção.

Com essa aplicação foi possível modelar as relações de dependências e feedback entre os critérios de seleção, além de permitir o ordenamento (ranking) dos critérios propiciando aos gestores uma forma de organização e estruturação de suas preferências, mostrando-lhes quais critérios são mais relevantes para a seleção de fornecedores.

Analisando o ranking apresentado na Figura 5 mostrou-se que a utilização de um 
método de ordenação (como o ANP), na etapa de pré-qualificação, pode levar a seleção de fornecedores que não atendam aos requisitos mínimos de desempenho em algum dos critérios, por permitir a compensação entre o desempenho dos critérios.

Por este motivo, utilizou-se da abordagem ratings, definindo níveis de intensidade mínimos de desempenho em cada critério, de forma a eliminar os fornecedores que não atendem aos requisitos mínimos de desempenho.

Uma vantagem da utilização da abordagem ratings é a possibilidade de inserir e remover alternativas durante o processo decisório, sem ocasionar prejuízo na estrutura e sem ocasionar inversão de ranking, além de permitir a análise de uma quantidade maior de alternativas (fornecedores), quando comparado com o ANP tradicional.

\section{CONCLUSÕES}

O SSP é constituído por cinco etapas: 1) Definição do problema; 2) Formulação dos critérios de decisão; 3) Pré-qualificação dos fornecedores potenciais; 4) Seleção final dos fornecedores e 5) Monitoramento dos fornecedores selecionados. Este trabalho propôs a aplicação do método ANP com a abordagem ratings na etapa de pré-qualificação dos fornecedores potenciais (etapa 3 ).

$\mathrm{Na}$ literatura essa etapa é abordada tanto com técnicas recomendadas para a classificação ou ordenação. Neste trabalho foi abordado com um método de ordenação.

A utilização do método ANP com a abordagem ratings pode levar a escolha de alternativas que apresentam desempenho baixo ou muito baixo em algum critério (nó), como já previsto devido a característica do método de compensação entre os desempenhos dos critérios.

Entretanto, com a definição de um desempenho mínimo aceitável em cada critério (nó), denominado limiar neste trabalho, foi possível identificar os fornecedores que não deveriam ser pré-qualificados na etapa 3.

Apesar de DeBoer et al. (2001) sugerir o uso de métodos de classificação para a etapa 3, este trabalho mostrou que é possível utilizar o método ANP com abordagem ratings, destacando-se a necessidade de utilizar limares nos critérios (desempenho mínimo) para lidar com este tipo de problema de forma mais adequada. Ou seja, a utilização de um método compensatório, sem limiares, para a etapa de pré-qualificação de fornecedores poderia selecionar fornecedores que não atendam aos requisitos mínimos de desempenho em algum dos critérios.

Para trabalho futuro, pretende-se estruturar o problema decisório com a abordagem Value-Focused Thinking (VFT) ou Value-Focused Brainstorming (VFB) para a definição dos critérios; assim como abordar a etapa 4 (seleção final dos fornecedores) com o método ANP integrado com a abordagem fuzzy (SIMIC et al., 2017).

\section{AGRADECIMENTOS}

Este trabalho foi parcialmente apoiado pelo Conselho Nacional de Desenvolvimento Científico e Tecnológico - CNPq.

\section{REFERÊNCIAS BIBLIOGRÁFICAS}

[1] AISSAOUI, N.; HAOUARI, M.; HASSINI, E. Supplier selection and order lot sizing modeling: a review. Computers \& Operations Research, v. 34, n. 12, p. 3516-3540, 2007. 
[2] AGARWAL, P.; SAHAI, M.; MISHRA, V.; BAG, M.; SINGH, V. A Review of MultiCriteria Decision Making Techniques for Supplier Evaluation and Selection. International Journal of Industrial Engineering Computations. Growing Science Publication, v. 2, n. 4, p. 801-810, 2011.

[3] ASSELlAOU, H.; OUHBI, B.; FRIKH B. Supplier Selection problem: a mathematical models story. In: 3rd INTERNATIONAL CONFERENCE ON LOGISTICS OPERATIONS MANAGEMENT (GOL), Fez, Morocco, 2015.

[4] ARAZ, C.; OZKARAHAN, I. Supplier evaluation and management system for strategic sourcing based on a new multicriteria sorting procedure. International Journal of Production Economics, v. 106, n. 2, p. 585-606, 2007.

[5] BHUTTA, M. K. S. Supplier selection problem: methodology literature review. Supply Chain Management: An International Journal, v. 7, n. 3, p. 126-135, 2003.

[6] BRUNO, G.; ESPOSITO, E.; GENOVESE, A.; PASSARO, R. The Analytical Hierarchy Process in the supplier selection problem. In: 10th INTERNATIONAL SYMPOSIUM ON THE ANALYTIC HIERARCHY/NETWORK PROCESS, Pittsburgh. ISAHP, 2009.

[7] CHAI, J.; LIU, J.N.K.; NGAI, E.W.T. Application of decision-making techniques in supplier selection: a systematic review of literature. Expert Systems with Applications, v. 40, n. 10, p. 3872-3885, 2013.

[8] DEBOER, L.; LABRO, E.; MORLACCHI, P. A review of methods supporting supplier selection. European Journal of Purchasing and Supply Management, v. 7, n. 2, p. 75-89, 2001.

[9] DICKSON, G. W. An analysis of vendor selection systems and decisions. Journal of Purchasing, v. 2, n. 1, p. 5-17, 1966.

[10] GALO, N. R.; CARPINETTI, L. C. R. Método de apoio à tomada de decisão em grupo para elicitação de preferências e categorização de fornecedores baseado em Fuzzy Delphi, Hesitant Fuzzy e ELECTRE TRI. In: SIMPÓSIO BRASILEIRO DE PESQUISA OPERACIONAL, 50,, 2018, Rio de Janeiro, RJ. Anais [...]. Rio de Janeiro: PUC-Rio, 2018.

[11] GENOVESE, A.; LENNY KOH, S. C.; BRUNO, G.; ESPOSITO, E. Greener Supplier Selection: state of the art and some empirical evidence. International Journal of Production Research, v. 51, n. 10, p. 2868-2886, 2013.

[12] GOVINDAN, K.; RAJENDRAN, S.; SARKIS, J.; MURUGESAN, P. Multi-criterion decision making approaches for green supplier evaluation and selection: a literature review. Journal of Cleaner Production, v. 98, p. 66-83, 2015.

[13] HAMDI, F.; GHORBEL, A.; MASMOUDI, F.; DUPONT, L. Optimization of a supply portfolio in the context of supply chain risk management: literature review. Journal of Intelligent Manufacturing, v. 29, n. 4, p. 763-788, 2015.

[14] HO, W.; XU, X.; DEY, P. Multi-criteria decision making approaches for supplier evaluation and selection: a literature review. European Journal of Operational Research, v. 202, n. 1, p. 16-24, 2010. 
[15] LIMA JUNIOR, F. R.; OSIRO, L.; CARPINETTI, L. C. R. A fuzzy inference and categorization approach for supplier selection using compensatory and noncompensatory decision rules. Applied Soft Computing Journal, v. 13, n. 10, p. 41334147, 2013.

[16] OCAMPO, L.A.; ABAD, G.K.M.; CABUSAS, K.G.L.; PADON, M.L.A.; SEVILLA, N.C. Recent approaches to supplier selection: a review of literature within 2006-2016. International Journal Integrated Supply Management, v. 12, n. 1-2, p. 22-68, 2018.

[17] SAATY, T. L. Rank from comparisons and from ratings in the analytic hierarchy/network processes. European Journal of Operational Research, v. 168, n. 2, p. 557-570, 2006.

[18] SAATY, T. L.; OZDEMIR, M. The Encyclicon a dictionary of applications of decision making with dependence and feedback based on the analytic network process. Pittsburgh: RWS Publications, 2005. 292 p.

[19] SARKAR, A.; MOHAPATRA, P. K. J. Evaluation of supplier capability and performance: A method for supply base reduction. Journal of Purchasing and Supply Management, v. 12, n. 3, p. 148-163, 2006.

[20] SEPULVEDA, J. M.; DERPICH, I. S. Multicriteria supplier classification for DSS: Comparative analysis of two methods. International Journal of Computers, Communications and Control, v. 10, n. 2, p. 238-247, 2015.

[21] SILVA, Amanda Cecília Simões. Utilização de Abordagens Não Usuais na Tomada de Decisão Multicritério à Solução do Problema de Seleção de Fornecedores. 2012. Tese (Doutorado em Engenharia Aeronáutica e Mecânica) - Instituto Tecnológico de Aeronáutica (ITA), São José dos Campos, 2012.

[22] SIMIC, D.; KOVACEVIC, I.; SVICEVIC, V.; SIMIC, S. 50 years of fuzzy set theory and models for supplier assessment and selection: A literature review. Journal of Applied Logic, v. 24 (Part A), p. 85-96, 2017.

[23] SONMEZ, M. A review and critique of supplier selection process and practices. Business School Occasional Papers Series, v. 1, n. 1, p. 1-34, 2006.

[24] WARE, N. R., SINGH, S. P., BANWET, D. K. (2012). Supplier Selection Problem: A state-of-the-art review. Management Science Letters, 2:1465-1490.

[25] WetZSTEIN, A.; HARTMANN, E.; BENTON JR, W. C.; HOHENSTEIN, N. O. A systematic assessment of supplier selection literature - State-of-the-art and future scope. International Journal Production Economics, v. 182, p. 304-323, 2016.

[26] YILDIZ, A.; YAYLA, A. Y. Multi-criterion decision making methods for supplier selection: a literature review. Journal of Industrial Engineering, v. 26, n. 2, p. 158$177,2015$. 